\title{
High-precision metrology of highly charged ions via relativistic resonance fluorescence
}

\author{
O. Postavaru, Z. Harman ${ }^{*}$ and C. H. Keitel \\ Max Planck Institute for Nuclear Physics, Saupfercheckweg 1, 69117 Heidelberg, Germany and \\ ExtreMe Matter Institute EMMI, Planckstrasse 1, 64291 Darmstadt, Germany
}

\begin{abstract}
Resonance fluorescence of laser-driven highly charged ions is studied in the relativistic regime by solving the time-dependent master equation in a multi-level model. Our ab initio approach based on the Dirac equation allows for investigating highly relativistic ions, and, consequently, provides a sensitive means to test correlated relativistic dynamics, bound-state quantum electrodynamic phenomena and nuclear effects by applying coherent light with x-ray frequencies. Atomic dipole or multipole moments may be determined to unprecedented accuracy by measuring the interferencenarrowed fluorescence spectrum.
\end{abstract}

PACS numbers: 78.70.En,31.30.Jv,32.70.Jz,31.30.Gs

High-precision laser spectroscopy has resulted in crucial advancements in our understanding of nature. In particular, optical laser spectroscopy (OS) is a versatile tool to investigate correlated relativistic quantum dynamics, the testing of fundamental theories like quantum electrodynamics (QED) 1, 2 or parity non-conservation in atomic systems. The determination of atomic dipole or multipole moments via lifetime measurements by means of, e.g., visible emission spectroscopy [3], approaching the accuracy of one per thousand, sheds light on QED effects like the electron anomalous magnetic moment. Isotope shifts (IS) in atomic spectra which has been providing valuable insight into the collective structure of nuclei: for example, recently, isotope shifts were determined by collinear laser spectroscopy [4. Beyond purely nuclear effects, the interaction of the correlated motion of electrons and that of the nucleus can be studied in IS measurements: recently, relativistic effects on nuclear recoil have been measured in visible forbidden transitions of few-electron argon ions by a trapped-ion method [5].

In the regime of heavy few-electron systems, however, the accuracy of optical spectroscopy can seldom be exploited due to the scarcity of low-frequency transitions. Therefore, one has to apply other techniques. Measuring x-ray emission lines of highly charged uranium ions confined in an electron beam ion trap allowed testing highfield QED on the two-loop level [1] and delivered a new value for the radius of the radioactive isotope ${ }^{235} \mathrm{U}[6]$. Recently, a method based on the storage ring measurement of dielectronic recombination spectra yielded the change of charge radii for neodymium isotopes [7, 8].

With the advent of modern short-wavelength laser systems, the accuracy and versatility of laser spectroscopy may be combined with the increased sensitivity of highly charged ions (HCI) to relativistic and QED effects, nuclear properties, and this may also give new information on HCI structure and dynamics relevant in astrophysical and thermonuclear plasmas. Brilliant x-ray light has already enabled to study transitions in the soft $\mathrm{x}$-ray regime involving HCI 9]. Coherent light with photon energies over $10 \mathrm{keV}$ becomes accessible in the near fu- ture [10, allowing for an extension to heavier systems and the exploitation of coherence properties. This would also ask for the validity of numerous quantum control schemes of resonance fluorescence [11 19] for concrete systems in the relativistic regime.

In the present Letter, we investigate the possibility of measuring atomic transition dipole - or multipole - moments and transition energies via relativistic resonance fluorescence of a three-level atomic configuration driven by two fields, namely, a short-wavelength laser and a long-wavelength light source in the optical regime. In such a three-level setting, the linewidth of the spontaneous transition of interest may be rendered much narrower than the natural linewidth, with the simultaneous increase of the total emitted intensity by orders of magnitude. Due to this effect, the determination of atomic multipole moments by means of the detection of the fluorescence spectrum is anticipated to increase in accuracy by several orders of magnitude.

As relativistic effects on the electronic wave function increase rapidly with the nuclear charge number $Z$, we formulate a fully relativistic ab initio theory of coherent laser-atom interaction based on the Dirac equation. An approach via the time-dependent numerical solution of this wave equation was recently employed to describe ionization phenomena [20. For our purposes, one needs to go beyond this approach and incorporate radiative relaxation in bound-bound transitions.

The interaction of a radiation field, having the fourvector potential $A_{\mu}(\mu \in\{0,1,2,3\})$, with a singleelectron atom can be described by the Hamiltonian (in relativistic units) $\mathscr{H}=\mathscr{H}_{A}+\mathscr{H}_{F}+e \gamma^{\mu} A_{\mu}$. Here, $\mathscr{H}_{A}$ and $\mathscr{H}_{F}$ are the energy operators of the atom and the radiation field, respectively, $e$ is the elementary charge, and $\gamma^{\mu}$ is the four-vector of Dirac matrices, represented in terms of $2 \times 2$ Pauli matrices. The atom is described in a fully relativistic manner, i.e. $\mathscr{H}_{A}=i \gamma^{\mu} \partial_{\mu}+V(r)$, where $V(r)$ is the nuclear potential, $r$ the distance from the origin, and $\partial_{\mu}$ is the space-time gradient operator. The Hamiltonian of the electromagnetic field $\mathscr{H}_{F}$ is given in terms of the photon creation and annihilation operators 
by $\mathscr{H}_{F}=\sum_{\mathbf{k}} \omega_{k}\left(a_{\mathbf{k}}^{\dagger} a_{\mathbf{k}}+\frac{1}{2}\right)$. To put the total Hamiltonian in a more convenient form, $\mathscr{H}_{A}$ and $\gamma^{\mu} A_{\mu}$ can be expressed in terms of the atomic operators $\sigma_{a b}=|a\rangle\langle b|$ describing transitions between a state $|a\rangle$ and $|b\rangle(a, b \in \mathbb{N})$. Let us introduce the coupling constants (Rabi frequencies) $g_{a b}=-\boldsymbol{\mu}_{a b} \cdot \epsilon_{\mathbf{k}} \mathscr{E} \mathbf{k}$, proportional to the corresponding laser field strength, with the relativistic multipole transition matrix element $\boldsymbol{\mu}_{a b}=\frac{e}{\omega}\left\langle a\left|\boldsymbol{\alpha} \cdot \epsilon_{\mathbf{k}} e^{i \mathbf{k} \cdot \mathbf{r}}\right| b\right\rangle$. Here, $\epsilon_{\mathbf{k}}$ denotes the laser polarization, $\omega=|\mathbf{k}|$ and $\mathscr{E}_{\mathbf{k}}$ is the amplitude of the electric field. We evaluate the multipole transition matrix elements by using the multipole expansion of the vector potential

$$
\epsilon_{\mathbf{k}} e^{i \mathbf{k} \cdot \mathbf{r}}=4 \pi \sum_{J M \lambda} i^{J-\lambda}\left(\mathbf{Y}_{J M}^{(\lambda)}(\hat{k}) \cdot \epsilon_{\mathbf{k}}\right) \mathbf{a}_{J M}^{(\lambda)}(\mathbf{r})
$$

The electric $(\lambda=1)$ and magnetic $(\lambda=0)$ multipole potentials $\mathbf{a}_{J M}^{(\lambda)}(\mathbf{r})$ are given by

$$
\begin{aligned}
\mathbf{a}_{J M}^{(0)}(\mathbf{r}) & =j_{L}(k r) \mathbf{Y}_{J L M}(\hat{r}), \\
\mathbf{a}_{J M}^{(1)}(\mathbf{r}) & =\sqrt{\frac{J+1}{2 J+1}} j_{J-1}(k r) \mathbf{Y}_{J J-1 M}(\hat{r}) \\
& -\sqrt{\frac{J}{2 J+1}} j_{J+1}(k r) \mathbf{Y}_{J J+1 M}(\hat{r})
\end{aligned}
$$

in the transverse gauge, which can be regarded as a relativistic generalization of the velocity form. The symbols $\mathbf{Y}_{J M}^{(\lambda)}(\hat{r})$ and $\mathbf{Y}_{J L M}(\hat{r})$ stand for the vector spherical harmonics. We adopt the generalized length gauge, also referred to as the Babushkin gauge [21. This general description also accounts for higher-order (non-dipole) transitions resulting in narrow spectral features. The Hamiltonian accounting for the interaction of the threelevel system with two classical fields is [11]

$$
\begin{aligned}
\mathscr{H}_{I} & =g_{31}\left(e^{i \Delta_{x} t} \sigma_{31}+e^{-i \Delta_{x} t} \sigma_{13}\right) \\
& +g_{21}\left(e^{i \Delta_{o} t} \sigma_{21}+e^{-i \Delta_{o} t} \sigma_{12}\right),
\end{aligned}
$$

where $\Delta_{x}=\omega_{31}-\omega_{x}$ and $\Delta_{o}=\omega_{21}-\omega_{o}$ are the detunings of the x-ray and optical laser frequencies $\omega_{x}, \omega_{o}$ from the atomic transition energies. The master equation describing the dynamics of the system with the Hamiltonian in the interaction picture is given by $\dot{\rho}=-i\left[\mathscr{H}_{I}, \rho\right]+\Lambda \rho$, where $\Lambda$ is the Lindblad operator accounting for the relaxation channels [11, 17 19]. We have also included the dephasing rate $\gamma_{D}$ of x-ray lasers by adding it to the decay rate $\gamma_{13}=\frac{1}{2}\left(\Gamma_{31}+\Gamma_{32}\right)$ of the $3 \leftrightarrow 1$ x-ray transition [17, 22, since the coherence time of free electron lasers (FELs) is often shorter than their pulse length. $\Gamma_{a b}$ denotes the partial radiative rate for the $\mathrm{a} \rightarrow \mathrm{b}$ transition.

Our goal is to evaluate the power spectrum of radiation scattered by a few-level atom driven by an incident field of arbitrary strength. To this end we introduce the field operators $\mathbf{E}^{( \pm)}(\mathbf{r}, t)$ for a given time $t$ and observation point $\mathbf{r}$ in the Weisskopf-Wigner approximation

$$
\mathbf{E}^{(+)}(\mathbf{r}, t)=\frac{\omega^{2} \sin \eta}{4 \pi r} \hat{e}_{x} P^{(+)}(t-r),
$$

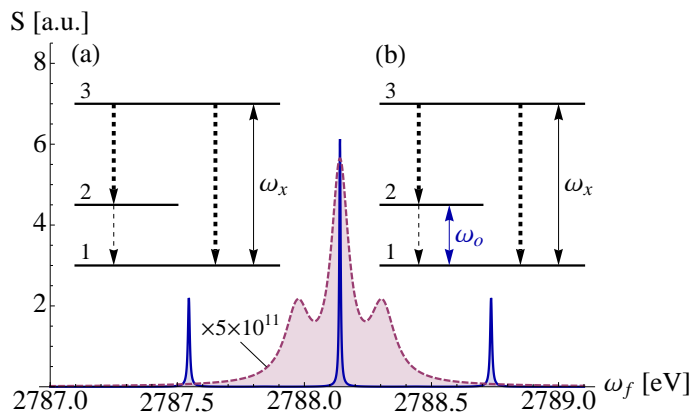

FIG. 1: (Color online.) Fluorescence photon spectrum for the $2 s \leftrightarrow 2 p_{3 / 2}$ transition in Li-like ${ }^{209} \mathrm{Bi}$ as a function of the fluorescence photon frequency $\omega_{f}$. (a) Dashed (red) curve: An x-ray laser $\left(I_{x}=5 \times 10^{11} \mathrm{~W} / \mathrm{cm}^{2}\right)$ is in resonance with the ionic electric dipole (E1) transition at $\omega_{31}=2788.1 \mathrm{eV}$ between the hyperfine-split ground state 1 ( $2 s$ with $F=4$, $\left.M_{F}=4\right)$ and the uppermost state $3\left(2 p_{3 / 2}\right.$ with $F=5$, $\left.M_{F}=5\right)$. This curve is multiplied by a factor of $5 \times 10^{11}$. Thick (thin) dashed arrows represent fast E1 x-ray (slow M1 optical) decays. (b) Continuous (blue) curve: an additional optical driving $\left(I_{o}=10^{14} \mathrm{~W} / \mathrm{cm}^{2}\right)$ is applied on the $\omega_{21}=$ $0.797 \mathrm{eV}$ 23] M1 transition between the hyperfine-split magnetic sublevels $1\left(F=4, M_{F}=4\right)$ and $2\left(F=5, M_{F}=5\right)$. The inner sidebands are suppressed. See text for more details.

and the corresponding conjugate expression for $\mathbf{E}^{(-)}(\mathbf{r}, t)$. Here, $P(\tau)^{(+)}=\boldsymbol{\mu}_{12}\left(\sigma_{12}+\sigma_{21}\right)+\boldsymbol{\mu}_{13}\left(\sigma_{13}+\right.$ $\left.\sigma_{31}\right)$. The atomic dipole (or, more generally, multipole) is assumed to be in the plane defined by the Cartesian coordinates $x$ and $z$, and $\eta$ is the angle between the dipole and the $z$-axis.

We are interested in the field emitted by the atom fixed in position along the $x$-axis. The field operator in Eq. (4) can therefore be treated as scalar. The power spectrum $S\left(\mathbf{r}, \omega_{0}\right)$ of the fluorescent light at some suitably chosen point $\mathbf{r}$ in the far field is obtained by taking the Fourier transform of the normallyordered field correlation function $\left\langle\mathbf{E}^{(-)}(\mathbf{r}, t) \mathbf{E}^{(+)}(\mathbf{r}, t+\tau)\right\rangle$ with respect to the time delay $\tau$. According to the Wiener-Khintchine theorem, the power spectrum $S\left(\omega_{0}\right)$ is $S\left(\omega_{0}\right)=\frac{1}{\pi} \operatorname{Re} \int_{0}^{\infty} d \tau\left\langle\mathbf{E}^{(-)}(\mathbf{r}, t) \mathbf{E}^{(+)}(\mathbf{r}, t+\tau)\right\rangle e^{i \omega_{0} \tau}$.

We calculate the power spectrum and the transition energies and matrix elements involved by relativistic methods. The Dirac wave functions corresponding to a rotationally symmetric nuclear potential are given in spherical coordinates in the form (see, e.g. 21] )

$$
\Phi_{a}(\boldsymbol{r})=\langle\boldsymbol{r} \mid a\rangle=\left(\begin{array}{c}
G_{n_{a} \kappa_{a}}(r) \Omega_{\kappa_{a} m_{a}}(\hat{r}) \\
i F_{n_{a} \kappa_{a}}(r) \Omega_{-\kappa_{a} m_{a}}(\hat{r})
\end{array}\right) .
$$

The subscript $a$ stands collectively for the principal quantum number $n_{a}$ as well as the angular momentum quantum numbers $j_{a}$ and $l_{a}$ and the magnetic quantum number $m_{a}$ of a given state $a \equiv\left(n_{a}, j_{a}, l_{a}, m_{a}\right)$. For Coulomb potentials, the bound radial functions $G_{a}(r)$ and $F_{a}(r)$ are known analytically, involving confluent hypergeometric functions with negative-integer first argument. The 
spin-angular part of the Dirac wave function is defined by the spherical spinors $\Omega_{\kappa_{a} m_{a}}(\hat{r})$ [21]. In the transition energies $\omega_{a b}$, also the one-loop QED corrections of selfenergy and vacuum polarization 24] have been included.

In Fig. 1 (a) we plot the power spectrum of resonance fluorescence for the case of the $2 s \leftrightarrow 2 p_{3 / 2}$ electric dipole transition in Li-like ${ }^{209} \mathrm{Bi}(Z=83)$ ions. The dynamic (AC) Stark shift leads to a splitting of the central peak, giving rise to a Mollow spectrum. Due to the long lifetime of the upper level of the hyperfine-split ground state, level 2 , almost $100 \%$ of the population is trapped in this level if only the $3 \leftrightarrow 1$ transition is driven coherently with an $\mathrm{x}$ ray laser. The calculation yields for the population of the uppermost state a value of approx. $\Gamma_{21} /\left(\Gamma_{32}+2 \Gamma_{21}\right) \approx$ $10^{-12} \ll 1$, resulting in a negligibly small total $\mathrm{x}$-ray fluorescence 25]. This undesirable effect may be reversed if additionally the $2 \leftrightarrow 1$ optical transition is coherently driven (see Fig. 1), leading to an efficient re-population of level 3.

Furthermore, the spectral lines become substantially narrower due to coherence and interference effects (see [11 for the pioneering non-specific treatment). The width of the central peak and the outer sidebands are given by $\Gamma_{C}=\left(\Gamma_{31}+\Gamma_{32}+\gamma_{D}\right) R+\Gamma_{21}(1-R)$ and $\Gamma_{S B}=\left|\frac{3}{2}\left(\Gamma_{31}-\frac{1}{3} \gamma_{D}\right) R+\frac{1}{2} \Gamma_{32}\left(R+R^{2}\right)+\frac{3}{2} \Gamma_{21}(1-R)\right|$, respectively, with the ratio $R$ being $g_{31}^{2} /\left(g_{31}^{2}+g_{21}^{2}\right)$ [25]. This effect is shown in Fig. 1 (b). Further increasing the intensity of the long-wavelength driving field and thus $g_{21}$ could even assign the narrow linewidth of $7.7 \cdot 10^{-15} \mathrm{eV}$ of the M1 hyperfine transition to the E1 x-ray transition of interest. The above line width formulas also imply that the dephasing width $\gamma_{D}$-typically on the order of $1 \mathrm{eV}$ for XFELs 10] - does not hamper the observation of subnatural linewidths in the x-ray regime as its contribution is decreased with the same factor $R$.

Transition lifetimes - and related quantities like the atomic dipole or multipole moments - are of great interest for astrophysical applications and for testing fundamental theories. Measurements of these quantities are particularly necessary since they are especially sensitive to the long-range behavior of atomic wave functions. Currently, even the best measurements do not exceed the $10^{-3}$ level of accuracy 3 . In our scheme, the narrowed central and outer lines enable in principle an even more accurate determination of the atomic Rabi frequencies: the outer sideband peaks' distance is given by $D_{s}(0)=4 G=4 \sqrt{g_{31}^{2}+g_{21}^{2}}$ (in the secular limit [25] and when the x-ray laser is on resonance, i.e. its detuning $\Delta=\omega_{31}-\omega_{x}$ from the transition frequency is 0 ). In this formula, the optical Rabi frequency $g_{21}$ is usually known, therefore, determining its counterpart $g_{31}$ for the x-ray transition is only limited by the accuracy of measuring the peak distance $D$. Fig. 2 (b) shows the ratio of the width of the narrowed outer lines to their distance $D_{s}(0)$. As shown, this ratio, characterizing the relative accuracy for the determination of atomic multipole moments, can
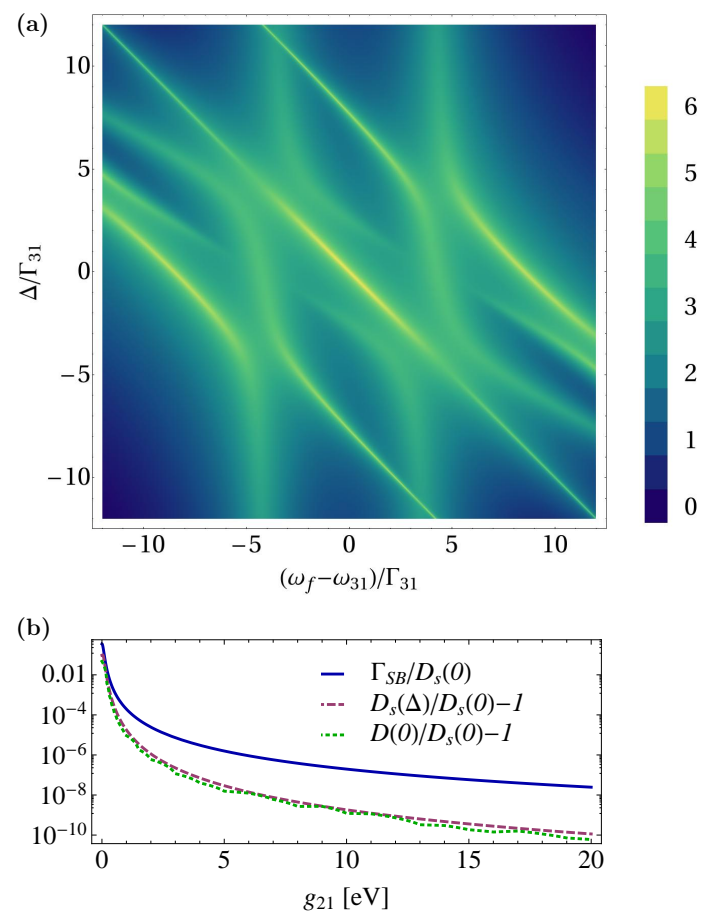

FIG. 2: (Color online.) (a) Density plot of the fluorescence spectrum (logarithmic scale, arb. units) as a function of the fluorescence photon frequency $\omega_{f}$ with respect to the x-ray transition frequency $\omega_{31}$ (abscissa) and the laser detuning $\Delta=\omega_{x}-\omega_{31}$ (ordinate), with the frequencies normalized by the $\Gamma_{31}$ rate. The parameters are for $\mathrm{Bi}$ as in Fig. 1. (b) Continuous (blue) curve: ratio of the interferencenarrowed width $\Gamma_{S B}$ of the outer sideband peaks to their distance $D_{s}(0)=4 G=4 \sqrt{g_{31}^{2}+g_{21}^{2}}$ as a function of the optical Rabi frequency $g_{21}$, with further parameters for the Bi threelevel system as given in the third line of TableI. Dashed (red) curve: deviation of the sideband distance $D_{s}\left(\Delta=\Gamma_{31}\right)$ from $D_{s}(0)$. Dotted (green) curve: deviation of the exact sideband distance $D(0)$ from its value in the secular limit $D_{s}(0)$.

be improved by several orders of magnitude for higher optical laser intensities.

Our calculation, in analogy with the derivation of Ref. [1] for zero detuning, shows that the outer sideband distance for non-zero detuning is given by $D_{s}(\Delta)=$ $4 G+\frac{G}{2}\left(4 R-3 R^{2}\right)(\Delta / G)^{2}+\mathcal{O}\left((\Delta / G)^{4}\right)$. This weak dependence is also illustrated in Fig. 2 (a). Hence, the experimental sensitivity on the potentially inaccurately known detuning may be reduced by orders of magnitude by increasing the optical intensity (Rabi frequency), as also shown in Fig. 2(b). The multipole matrix elements of the ionic transitions can thus be determined in principle to a high accuracy on the order of $10^{-4}-10^{-6}$, once the intensity of the driving field is accurately known. Conversely, the intensity may be measured to high accuracy if the multipole moments are reliably known from an independent experiment (e.g. lifetime measurements). At the same time, knowing the Rabi frequencies, the dependence of sideband positions on the x-ray detuning 
TABLE I: Parameters for $2 s \leftrightarrow 2 p_{3 / 2}$ transitions in the Lilike ions ${ }^{203} \mathrm{Tl}^{78+}\left(\omega_{31}=2236.5 \mathrm{eV}\right),{ }^{209} \mathrm{Bi}^{80+}(2788.1 \mathrm{eV})$ and ${ }^{235} \mathrm{U}^{89+}(4459.4 \mathrm{eV})$. Optical transition energies $\left(\omega_{21}, 23\right)$, natural line widths $\left(\Gamma_{31}, \Gamma_{21}\right)$ and Rabi frequencies $\left(g_{31}, g_{21}\right)$ as well as the interference-narrowed outer sideband width $\left(\Gamma_{S B}\right)$ of the x-ray transition are given for the laser intensities $I_{x}, I_{o} . x(y)$ stands for $x \times 10^{y}$.

\begin{tabular}{|c|c|c|c|c|c|c|c|c|}
\hline & $\omega_{21}$ & $\Gamma_{31}$ & $\Gamma_{S B}$ & $\Gamma_{21}$ & $g_{31}$ & $g_{21}$ & $I_{x}$ & $I_{o}$ \\
\hline & \multicolumn{6}{|c|}{$[\mathrm{meV}]$} & \multicolumn{2}{|c|}{$\left[\mathrm{W} / \mathrm{cm}^{2}\right]$} \\
\hline \multirow[t]{2}{*}{$\mathrm{Tl}$} & 499 & 6.6 & $7.1(-2)$ & $1.1(-12)$ & $1.8(2)$ & $2.1(3)$ & $1(12)$ & $1(16)$ \\
\hline & & & $7.2(-4)$ & & $1.8(2)$ & $2.1(4)$ & $1(12)$ & $1(18)$ \\
\hline \multirow[t]{2}{*}{$\mathrm{Bi}$} & 797 & $7.2(1)$ & $9.7(-2)$ & $7.7(-12)$ & $8.3(1)$ & $2.9(3)$ & $5(11)$ & $1(16)$ \\
\hline & & & $1.9(-1)$ & & $1.2(3)$ & $2.9(4)$ & $1(14)$ & $1(18)$ \\
\hline \multirow[t]{2}{*}{$\mathrm{U}$} & 136 & $2.4(1)$ & $3.7(-2)$ & $3.7(-14)$ & $7.7(1)$ & $2.8(3)$ & $5(11)$ & $1(16)$ \\
\hline & & & 1.3 & & $3.3(4)$ & $1.9(5)$ & $9(16)$ & $5(19)$ \\
\hline
\end{tabular}

could allow to measure in principle the ionic x-ray transition energy in an independent way.

Our above results have been demonstrated on the example of HCI with non-zero nuclear spins, i.e. when hyperfine splitting of the ground state occurs. However, certainly, the results may be applied to further three-level systems. For example, such configurations may also be prepared by applying (strong) external magnetic fields, which gives rise to a large Zeeman splitting of the groundstate level, addressable by long-wavelength coherent radiation such as masers (or even $\mathrm{CO}_{2}$ lasers). Furthermore, the results can be generalized to other physical systems with high transition energies, such as electromagnetic transitions in nuclei. In this setting, nuclear multipole moments and transition energies may in principle be determined by an independent method.

Laser systems with photon energies of up to a few $\mathrm{keV}$ (in the range of Li-like transitions) are presently available [26, 27, allowing to excite elements as heavy as U and observing emission lines of sub-natural linewidths. Upcoming laser facilities are expected to increase the frequency limit to the order of tens of keVs (e.g. [10]), permitting to directly address the most relativistic heavy $\mathrm{H}$-like systems or even nuclear transitions. Table $\mathrm{I}$ lists values for some elements and atomic transitions.

In summary, a fully relativistic ab initio theory of the bound dynamics of atomic systems in laser fields ranging to the x-ray domain has been developed. The bare atomic states are constructed from solutions of the Dirac equation. This approach allows for exploiting the sensitivity of inner-shell electrons to relativistic electron correlation, QED and nuclear effects in strong Coulomb fields. As a demonstrative example, a means to determine ionic transition multipole moments and frequencies via a threelevel configuration driven by an x-ray and an optical field has been put forward. Current or near-future laser systems are expected to increase the accuracy of multipole moment determinations from the current $10^{-3}$ level (via lifetime measurements) to the $10^{-4}$ range or better. Furthermore, the undesirable trapping of atomic population in a long-lived metastable state - naturally occurring in certain three-level systems - can be reversed by the scheme presented here. Other scenarios developed for the quantum control of non-relativistic resonance fluorescence emission [12 19] are anticipated to yield further improvement of detection and accuracy.

The authors acknowledge helpful conversations with Mihai Macovei and Jörg Evers. Supported by Helmholtz Alliance HA216/EMMI.

* harman@mpi-hd.mpg.de

[1] P. Beiersdorfer et al., Phys. Rev. Lett. 95, 233003 (2005).

[2] A. Gumberidze et al., Phys. Rev. Lett. 94, 223001 (2005).

[3] A. Lapierre et al., Phys. Rev. Lett. 95, 183001 (2005).

[4] W. Geithner et al., Phys. Rev. Lett. 101, 252502 (2008).

[5] R. Soria Orts et al., Phys. Rev. Lett. 97, 103002 (2006).

[6] S. Elliott, P. Beiersdorfer, and M. Chen, Phys. Rev. Lett. 76, 1031 (1996).

[7] C. Brandau et al., Phys. Rev. Lett. 100, 073201 (2008).

[8] R. Şchiopu et al., Eur. Phys. J. D. 31, 21 (2004).

[9] S. W. Epp et al., Phys. Rev. Lett. 98, 183001 (2007).

[10] European XFEL Project, http://xfel.desy.de/ technical_information/photon_beam_parameter/

[11] L. M. Narducci et al., Phys. Rev. A 42, 1630 (1990).

[12] S.-Y. Zhu and M. O. Scully, Phys. Rev. Lett. 76, 388 (1996).

[13] P. Zhou and S. Swain, Phys. Rev. Lett. 77, 3995 (1996).

[14] E. Paspalakis and P. L. Knight, Phys. Rev. Lett. 81, 293 (1998).

[15] M. O. Scully and S.-Y. Zhu, Science 281, 1973 (1998).

[16] J. Evers and C. H. Keitel, Phys. Rev. Lett. 89, 163601 (2002); M. Macovei and C. H. Keitel, Phys. Rev. Lett. 91, 123601 (2003).

[17] M. O. Scully and M. S. Zubairy, Quantum Optics (Cambridge University Press, Cambridge, 1997).

[18] Z. Ficek and S. Swain, Quantum Interference and Coherence (Springer, New York, 2005).

[19] M. Kiffner et al., Progress in Optics (2010).

[20] H. G. Hetzheim and C. H. Keitel, Phys. Rev. Lett. 102, 083003 (2009).

[21] I. P. Grant, Relativistic Quantum Theory of Atoms and Molecules (Springer, Berlin, Germany, 2006).

[22] G. S. Agarwal, Phys. Rev. Lett. 37, 1383 (1976).

[23] V. M. Shabaev et al., Phys. Rev. A 57, 149 (1998).

[24] P. Mohr, G. Plunien, and G. Soff, Phys. Rep. 293, 227 (1998).

[25] Our current discussion is valid in the secular limit $G=$ $\sqrt{g_{31}^{2}+g_{21}^{2}} \gg \max \left\{\Gamma_{31}, \Gamma_{32}, \Gamma_{21}\right\}$. If the effective Rabi frequency $G$ can not be rendered high enough for a sufficient applicability of this approximation, one has to work with the more accurate and lengthy expressions of the spectral features.

[26] V. Ayvazyan et al., Eur. Phys. J. D 37, 297 (2006).

[27] Stanford LCLS Specifications, https://slacportal. slac.stanford.edu/sites/lcls_public/Instruments/ SXR/Pages/Specifications.aspx 